\title{
Strafrecht und Verfassung
}

\author{
Gedanken nach dem 2. Symposium junger Strafrechtlerinnen und \\ Strafrechtler an der Humboldt-Universität zu Berlin
}

\author{
Jan C. Schuhr"
}

Ungefähr 100 Nachwuchswissenschaftler der „gesamten Strafrechtswissenschaft“ trafen sich vom 29. bis 31. März in Berlin. Es war das zweite Mal, dass die strafrechtliche Nachwuchstagung stattfand. ${ }^{1}$ Beatrice Brunböber, Tobias Reinbacher, Moritz Vormbaum (für die HU) sowie Katrin Höffler und Johannes Kaspar (beide noch für die LMU) hatten zu „Strafrecht und Verfassung“ an die Humboldt-Universität eingeladen.

Die Örtlichkeiten stimmen die Ankommenden in sehr eigener Weise auf die Auseinandersetzung mit dem Verfassungsrecht ein. Imposante Gebäude dokumentieren Selbstbewusstsein. Hier ist das deutsche Zentrum - „Mitte“. Macht, Stärke, schlichte und zugleich ostentative Eleganz stehen dem kleinen Menschen gegenüber, der sofort begreift, dass es höchstens indirekt auch um ihn geht. Er ist umgeben von raumgreifenden Gebäuden. Die Gegend ist weitläufig, und es weht ein kühler Wind. Der kleine Mensch mag etwas frieren, aber „Freiheit“ im Sinne von Platz, Luft und guter Himmelssicht sind ihm sicher wie sonst in Metropolen selten. Alles ist ehrwürdig. Die Architekten haben es so konstruiert. Ehre musste nicht erst den Unbillen vergangener Zeiten abgetrotzt und fortan behauptet werden. Nebenan ist eine große Baustelle, auf der etwas Altes entsteht. Auch an den fertigen Gebäuden wird gearbeitet. Wozu sie dienen, wäre kaum zu erkennen, gäben darüber nicht jeweils große, goldene Lettern Aufschluss. Erbaut wurde eine Ordnung. Sichtbar wird in ihr eine Haltung, während der Sinn akzidentiell bleibt. Eintreten und die Räume beleben muss jeder selbst. Innen ist es wärmer. Hier kann man merken, dass man doch willkommen ist, und es lässt sich gut gemeinsam arbeiten.

Der Call for Papers und die Einladung hatten Arzts Warnung vor einer Kolonialisierung des Strafrechts (GS Armin Kaufmann 1989, 839, 847 ff.) aufgegriffen und ihr Lagodnys Befund, das materielle Strafrecht entziehe sich in nahezu genialer Weise einer verfassungsrechtlichen Kontrolle (Strafrecht vor den Schranken der Grundrechte, 1996, S. 536), zur Seite gestellt. Die Thesen klingen, als stünden sie einander entgegen. Stehen sie aber nicht. Arzt betonte gerade, dass Kolonialisierung Stärke des Mutterlandes und Schwäche der Kolonie voraussetzt. Versuche, dem materiellen Strafrecht einen verfassungsrechtlichen Begriff der Strafe zu oktroyieren und das ganze System an der Rechtsfolge ansetzend ins Verfassungsrecht zu transferieren, sah

\footnotetext{
* Dr. Jan C. Schuhr ist Akad. Rat a.Z. am Lehrstuhl für Strafrecht, Strafprozessrecht und Rechtsphilosophie in Erlangen (Prof. Dr. Hans Kudlich).

1 Zum ersten Symposium in Köln vom 19. bis 20. November 2010 siehe Kaspar, RW 2011, 103 ff.
} 
er als erhebliche, aber bis dato weitgehend erfolgreich abgewehrte Gefahr an. Er beklagte den Verlust eines über längere Zeit vernachlässigten Strafprozessrechts an das Verfassungsrecht.

Die Bilder der Kolonialisierung, der Kontrolle und des Sich-Entziehens zeichnen eine offene Konfrontation von Verfassungsrecht und Strafrecht. Gleichwohl hätte sicherlich jeder Tagungsteilnehmer die Klarstellung unterschrieben, zu der sich schon Arzt veranlasst sah: „Ich freue mich über die Stärke des Verfassungsrechts und bin entschieden für ein verhältnismäßiges und überhaupt rechtsstaatliches Strafprozessrecht. "Unbestreitbar geht das Verfassungsrecht dem einfachen Recht, also auch dem Strafrecht und Strafprozessrecht, vor. Unbestreitbar müssen gerade das Strafrecht und Strafprozessrecht die Grundrechte und die Würde der Einzelnen achten und sichern und den Rechtsstaat Wirklichkeit werden lassen. Genau das gebietet das Grundgesetz. Und doch witterten die meisten Vortragenden und Diskutanten aus dem Verfassungsrecht nicht nur Gutes.

Ein Teil dieser Bedenken beruht auf überzogenen Erwartungen. Das arbeitete Luís Greco (LMU) in einer Gegenüberstellung von verfassungskonformem und legitimem Strafrecht heraus. Er beschrieb die Entwicklung vom Grundrechtsoptimismus und der Ausarbeitung der Grundrechtsdogmatik in der jungen Republik hin zum Verfassungsgerichtspositivismus, d.h. einer argumentativen Verselbständigung der vom Verfassungsgericht gegebenen Interpretationen. Auch Strafrechtler hegen immer wieder die Hoffnung, das BVerfG werde ihren Thesen eine erhöhte Verbindlichkeit verleihen. Wer seine Argumentation danach ausrichtet, missachtet indes oft Unterschiede in den Erkenntnisinteressen: Wo es dem Verfassungsrecht um die Grenze geht, ab der sich rechtswidriges Verhalten der Staatsgewalt zur Verfassungswidrigkeit steigert, muss das Strafrecht aufzeigen, wie die Strafsache optimal zu entscheiden wäre. Auch Hoffnungen auf eine Vereinfachung und Verwissenschaftlichung der Diskussion und einen Gewinn an Stringenz durch Bezugnahme auf das Verfassungsrecht setzte Greco grundsätzliche Zweifel entgegen: Soweit das Verfassungsrecht solche Vorteile zu vermitteln vermag, geschieht das durch formale, insbesondere normhierarchische Anforderungen. Dem Strafrecht muss es aber um materiale Inhalte und Stringenz gehen, die die Verfassung nicht vermittelt, sondern voraussetzt.

Die Verfassung bzw. die Verfassungslehre liefert in der strafrechtlich vor allem interessierenden Grundrechts- und Rechtsstaatsdogmatik eine Ordnung und eine Haltung. Das umfasst weit mehr als ein Prüfschema. Auch Inhalte gehören dazu, insbesondere die Schutzbereiche der Grundrechte (wobei der konkrete Inhalt der Grundrechte jeweils Gegenstand aller betroffenen Rechtsgebiete und nicht allein der Grundrechtsdogmatik ist). Die strafrechtlichen Inhalte und Konsistenzanforderungen aber sind grundrechtsdogmatisch meist Schranken der Grundrechte bzw. die Herstellung praktischer Konkordanz zwischen Grundrechten. Das gibt ihnen verfassungsrecht- 
lich nur eine Form. Inhalte setzt das Verfassungsrecht insoweit lediglich dort, wo es die Beschränkung bzw. Grenzziehung für unzulässig erklärt - und das ist (zu Recht) seltene Ausnahme. Ansonsten erkennt das Verfassungsrecht lediglich die Zulässigkeit und evtl. auch Notwendigkeit einer Einschränkung bzw. Grenzziehung an, überlässt diese (und damit die materiellen Probleme) aber „dem einfachen Recht“. Hierarchisch mag man dieses dann als „Ausführungsgesetz“ einordnen. Inhaltlich aber ordnet sich die Verfassung ihm unter und hat dazu auch gar keine Alternative, wenn nicht jede materielle Bestimmung der Rechtsordnung Verfassungsrang erhalten und die hierarchische Gliederung damit in sich zusammenfallen soll.

Selbst die eher seltene Ausnahme, dass eine konkrete strafrechtliche Regel - sie kann im Gesetz stehen oder in seiner Handhabung liegen - dem Verdikt der Verfassungswidrigkeit anheimfällt, liefert keinen positiven Inhalt (sondern ist nur als negative Grenzziehung inhaltlich). Wie die strafrechtliche Regel richtigerweise zu formulieren bzw. zu handhaben wäre, ergibt sich nicht mit den Methoden des Verfassungsrechts. Anders wäre dies nur, wenn der Verfassung konkrete Bestrafungsgebote entnommen werden könnten. Dass dies nicht der Fall ist, hat Milan Kubli (Frankfurt a.M.) eingehend dargestellt. Er legte dar, dass entgegen der wohl h.M. kollidierendes Verfassungsrecht grundsätzlich auch die Garantie des Art. 103 Abs. 2 GG beschränken könnte, indes nur ein zwingendes Bestrafungsgebot eine derartige Kollision auslösen würde. Doch ein solches Gebot besteht nicht einmal für die im Völkerstrafrecht behandelten schwersten Menschenrechtsverletzungen (dessen ungeschriebene Regeln das Schriftlichkeitsgebot nicht erfüllen).

Die „verfassungsrechtlichen Bauwerke“ sind aus Perspektive des Strafrechts also erst einmal „weitgehend leer“. Das bedeutet nicht, dass es verfassungsdogmatisch gleichgültig wäre, ob und wie sie gefüllt werden. Die Herstellung praktischer Konkordanz der Grundrechte ist ein Gebot der Verfassung. Sie wäre allerdings auch ohne die Verfassung geboten. Kant etwa erhebt die Abgrenzung der Freiheitssphären sogar zum „allgemeinen Prinzip des Rechts“ (AA VI S. 230). Ohne das Gebot der Verfassung müsste sie an gleicher Stelle in der Rechtsordnung erfolgen. Dem Strafrecht und Strafprozessrecht (neben anderen Rechtsgebieten) fiele also auch ohne das Grundgesetz genau die Aufgabe zu, die das Grundgesetz ihnen zuschreibt. Die Verfassung verschafft dem Gebot, praktische Konkordanz der Berechtigungen herzustellen, „nur“ einen besonderen Rang.

Entsprechende und ähnliche Gebote können sich aus der Verfassung auch an anderer Stelle ergeben. Ein Beispiel dafür hat Denis Basak (Frankfurt a.M.) anhand der Untreue-Entscheidung des BVerfG aus dem Jahr 2010 näher untersucht. Dort wurde Art. 103 Abs. 2 GG neben den klassischen vier Ausformungen von nulla poena sine lege (praevia, scripta, stricta et certa) ein an die Rechtsprechung gerichtetes Präzisierungsgebot entnommen. Das ist nicht nur eine Anforderung an strafrechtliche In- 
halte, sondern ein Gebot zur Schaffung strafrechtlicher Inhalte. Basak hat vor allem damit verbundene Probleme behandelt. Das Präzisierungsgebot kommt gerade dann zum Tragen, wenn noch keine hinreichend bestimmte Regelung besteht. Wird sie gleichwohl für verfassungskonform erklärt, entsteht immer wieder das Missverständnis, der Status quo werde dadurch goutiert. Mit der Verneinung der Verfassungswidrigkeit stellt das BVerfG indes keineswegs die Rechtmäßigkeit des Zustands fest - im Gegenteil: das Gebot, die Regelung weiter zu präzisieren, besteht gerade deshalb, weil sie den an sie zu stellenden Anforderungen noch nicht genügt.

Damit der Bedarf nach Inhalten - den das Verfassungsrecht selbst wahrnimmt, aber nicht befriedigt - gedeckt wird, enthält das Verfassungsrecht Zuständigkeitszuweisungen. Zentral sind dabei das Demokratie- und das Gewaltenteilungsprinzip. Beide liefern nicht nur grundsätzlich überzeugende Antworten, sondern führen in zahlreichen Details zu erheblichen Schwierigkeiten. Zunächst besteht ein Spannungsverhältnis zwischen Mehrheitsentscheidungen und dem Schutz von Individualrechten, insbesondere Grundrechtspositionen. Daneben ist die Feststellung, eine unterbestimmte Norm sei nicht verfassungswidrig unbestimmt, müsse aber von der Rechtsprechung weiter präzisiert werden, ambivalent. Einerseits sind sprachliche Unschärfen nie ganz zu beheben; entsprechende Anforderungen wären sinnlos. Zudem bestimmt eine unscharfe Grenzziehung nur innerhalb ihres Unschärfebereichs keine Grenze, sehr wohl aber außerhalb ihrer Unschärfe und kann dort sehr wertvoll sein. Andererseits steht die Präzisierungskompetenz der Rechtsprechung aber in einem Spannungsverhältnis zum Gewaltenteilungsprinzip, soweit das Gesetzlichkeitsprinzip die Schaffung einer hinreichend bestimmten Regelung gerade dem Gesetzgeber zuweist. Die Präzisierungslösung korrigiert eine „naive“, weil praktisch uneinlösbare und vor allem justiziell nicht durchsetzbare Kompetenzverteilung, indem sie Zuständigkeiten vom Gesetzgeber auf die Rechtsprechung verschiebt.

Der Bestimmtheitsgrundsatz spielt sogar in die Verteilung der Gesetzgebungskompetenz hinein. Erol Poblreich (HU) diskutierte zustimmend die Haltung des BVerfG, wonach die neue Zuständigkeitsverteilung der Föderalismusreform I von 2006 für den Vollzug von Straf- und Untersuchungshaft vom Bestimmtheitsgrundsatz insofern überlagert wird, als der Bundesgesetzgeber auch Vollzugsfragen in Grundzügen zu regeln hat, wenn er neue Sanktionstypen schafft; der Kern der Sanktion bliebe sonst unklar (vgl. BVerfGE 109, 190; 128, 326). Das kann zu nicht ganz unerheblichen Einschränkungen der eigentlich intendierten Länderzuständigkeit führen.

Hauptsächlich liefert Art. 103 Abs. 2 GG - idealistisch verklärend ausgedrückt - eine verfassungsgerichtliche Handhabe zur Durchsetzung des strafrechtlichen Gesetzlichkeitsprinzips bzw. - der praktischen Anwendung näher - Topoi und Rechtsfolgen, mit denen das BVerfG bei als besonders störend empfundener Missachtung des Gesetzlichkeitsprinzips oder als besonders dringlich angesehenem Regelungsbedarf 
steuernd eingreifen kann. Dass die strafrechtliche Lehre und gelegentlich Gerichte sich dabei mehr Rückendeckung gegenüber dem Gesetzgeber erhoffen würden, während sie Eingriffen des BVerfG in die eigene dogmatische Arbeit recht kritisch gegenüberstehen, ist kein Geheimnis. Dorothea Magnus (Univ. Hamburg) hat dies vorgeführt in einem Rückblick auf die Entwicklung des Gewaltbegriffs zu $\mathbb{} 240$ Abs. 1 StGB in der Strafrechtslehre, der fachgerichtlichen Rechtsprechung und Entscheidungen des BVerfG.

Tatsächlich sprechen gute Gründe gegen eine allzu enge Bindung der Strafrechtslehre an gesetzliche Formulierungen. Der parlamentarische Gesetzgeber ist dazu eingerichtet, Interessenkonflikte teils auszugleichen, teils zu entscheiden. Ein konsistentes Regelungsgefüge originär zu schaffen, übersteigt seine Möglichkeiten. Hier ist dogmatisches Vordenken erforderlich bzw. - soweit Widersprüche Gesetz geworden sind - dogmatisches Nachdenken mit dem Ziel ihrer Auflösung. In beiden Fällen entstehen die Inhalte in der dogmatischen Arbeit und nicht im Akt der Gesetzgebung. Dass gesetzgeberische Entscheidungen zu respektieren sind und in einer Demokratie Vorrang vor dogmatischen (und erst recht vor bloß rechtspolitischen) Wünschen haben, steht dem nicht entgegen, denn zu einem erheblichen Teil positivieren Parlamentsgesetze nur das dogmatisch Vorgedachte, ohne dass das Parlament dabei tatsächlich Interessenkonflikte entscheiden würde. Insoweit entscheidet es nur, dass die Fortschritte an Rechtsklarheit und Rechtssicherheit es rechtfertigen, den aktuellen Zwischenstand der dogmatischen Ausarbeitung zu kodifizieren und so für die Rechtspraxis fruchtbar zu machen. Es entspräche aber nicht dem Willen des Gesetzgebers, die Weiterarbeit an den halbausgereiften Gedanken zu verbieten. Die Bindung der Dogmatik an ein solches Gesetz liefe auf eine unsinnige Perpetuierung des Provisoriums und eine Selbstabschaffung der Wissenschaft hinaus.

Während das geltende Recht daher zumindest nicht als alleiniger Gegenstand der Rechtswissenschaft angesehen werden darf, gibt es keinen Freibrief, Arbeiten de lege lata und solche de lege ferenda beliebig zu vermengen. Die eigentlichen Probleme bestehen in Folgendem: Zunächst gilt es festzustellen, welche dogmatischen Änderungen im Gewand der „Präzisierung“ der bestehenden Rechtslage akzeptabel sind, um eine im Wesentlichen bestehende Kontinuität der Rechtslage zu dokumentieren. Soweit die Grenze der „bloßen Präzisierung “ überschritten ist, muss man herausfinden, wann Überlegungen de lege ferenda so weit ausgereift sind, dass ihre Positivierung besser wäre als ein Festhalten an der bestehenden Gesetzeslage. Schließlich fehlt ein Qualitätsmanagement für Gesetze, das dazu führen würde, dass in der Wissenschaft (und in der Rechtsprechung) erzielte Fortschritte systematisch in Gesetzesvorlagen aufgegriffen werden. Bislang geschieht das oft erst nach Jahrzehnten und abhängig von politischen Zufällen, die bei Interessenkonflikten einen guten Sinn haben können, nicht aber bei konfliktfreien bzw. konfliktarmen Regelungen. 
Ebenso wenig, wie der Verfassung die konkrete Austarierung der Grundrechte im Verhältnis zueinander zu entnehmen ist, steht in ihr, durch welche Verfahren der Rechtsstaat real wird, und die wenigsten konkreten Rechtspositionen, die er zu schützen hat, stehen in der Verfassung. Das zum materiellen Recht Gesagte gilt daher für das Verfahrensrecht meist in ähnlicher Weise. Oft sind die inhaltlichen Vorgaben dort noch schwächer, denn schon die Rechtsposition des Beschuldigten wird eher in Art. 6 EMRK skizziert als im Grundgesetz. Die ihr bei der Ausgestaltung des Verfahrens zur Seite oder entgegen zu stellenden Allgemeininteressen werden hingegen größtenteils nicht einmal als Topoi, geschweige denn mit Schutzbereichen, inneren Konflikten und Grenzen ausgearbeitet. Soweit das Verhältnismäßigkeitsprinzip es ermöglicht, „vernünftige Belange angemessen zu berücksichtigen“, ist es ein weit geratener Platzhalter für Inhalte, liefert aber kaum eine materielle Aussage.

Gefahren, die entstehen, wenn „die Subjektstellung des Beschuldigten“ und die „Rechtsstaatlichkeit und Fairness des Verfahrens“ nicht als Mahnung verstanden werden, sich an ausgearbeitete Verfahrensweisen zu halten und inhaltliche Bindungen zu entwickeln, hat Antje Schumann (Leipzig) am Beispiel der $\mathbb{S} 136,136$ a StPO analysiert. Diese Vorschriften enthalten Anforderungen an den Umgang mit dem Beschuldigten. Wenn die förmliche Vernehmung des Beschuldigten der einzige Weg ist, seine Mitteilungen in das Verfahren einzuführen, enthalten sie sogar einen erheblichen Teil seiner Rechtsposition, sind aber auch dann nicht abschließend. Sieht man sie hingegen als Kernverbürgung der Beschuldigtenrechte und beschränkt sie zugleich auf förmliche Vernehmungen, entsteht ein Regelungsvakuum. Die Ermittlungsbehörden sind dann grundsätzlich frei, auch auf andere Weise als durch Vernehmung „listig“ an Mitteilungen des Beschuldigten zu gelangen. Hinsichtlich dabei einzuhaltender Verfahren und der Rechtsposition des Beschuldigten fehlt aber die nähere Ausgestaltung. Die verfassungsrechtliche Rückbindung der $\mathbb{S} \mathbb{1 3 6}, 136$ a StPO suggeriert dann nur eine inhaltliche Vorgabe, wo tatsächlich weder im Verfassungsrecht noch im einfachen Recht konkrete Regelungen getroffen werden. Die Frage, ob das einfache Recht geronnenes Verfassungsrecht sein sollte, stellt sich so gar nicht; der Appell an Menschenwürde und Rechtsstaatlichkeit darf jedenfalls nicht das den Auftrag der Verfassung verfehlende Ausbleiben einer Regelung kaschieren.

Inhaltliche Vorgaben erhält das nationale Strafrecht zunehmend von anderer, „hierarchisch noch höherer" Stelle: Europarecht gewinnt an Bedeutung und beinhaltet sowohl Gebote zum Erlass von materiellem Strafrecht als auch zum Erlass verfahrensrechtlicher Bestimmungen mit inhaltlich sehr konkreten Vorgaben. Es gibt gute Gründe, in einem zusammenwachsenden Europa auch die Zusammenarbeit in Strafsachen zu intensivieren. Das dabei zentrale Prinzip der gegenseitigen Anerkennung von Entscheidungen lässt sich vielfach am besten umsetzen, indem Entscheidungsgrundlagen und Verfahren innerhalb Europas einander angeglichen werden. Gerade 
in Deutschland gibt es indes sowohl eine - in erheblichem Maße auf Unterschieden im Recht beruhende - weit verbreitete Skepsis gegenüber ausländischen Entscheidungen in Strafsachen, als auch einen beträchtlichen Argwohn gegenüber Rechtsangleichungen in diesem Bereich. Diesen Themenkreis behandelte Christina Globke (Mainz). Sie setzte sich mit dem Strafbegriff und an ihn anknüpfenden Rechtsprinzipien im Unionsrecht auseinander und stellte sie der deutschen Grundrechtsdogmatik gegenüber. Besondere Aufmerksamkeit widmete sie dabei der Lissabon-Entscheidung des BVerfG, in der das Gericht von einer „im Grundsatz integrationsfeste[n] mitgliedstaatliche[n] Strafkompetenz" ausgeht.

Die deutsche Strafrechtslehre nimmt ihr „Produkt“ als „Exportschlager“ wahr. Sie ist stolz auf die Aufnahme vor allem der deutschen Dogmatik des allgemeinen Teils z.B. in Ländern wie Spanien, Italien und Griechenland, aber auch außerhalb Europas bis in den Fernen Osten und Südamerika. Man kann sich über strafrechtliche Strukturen durchaus über nationale und kulturelle Grenzen hinweg verständigen. Für Straftatbestände gilt das zwar nur eingeschränkt, aber doch so weit, dass es eine generelle Ablehnung supranationalen Strafrechts nicht begründet. Auch dass - wie das BVerfG betont - die „demokratische Selbstgestaltungsfähigkeit eines Verfassungsstaates" gesichert werden muss, erfasst nicht das strafrechtlich Wesentliche. Die strafgesetzgeberische „Entscheidung über das rechtsethische Minimum“ ist sowohl wegen ihrer Eingriffsintensität in die Handlungsfreiheit als auch bei besonderer Eingriffsschwere der Rechtsfolgen höchst sensibel. Mehrheitsentscheidungen sichern keineswegs automatisch eine befriedigende Berücksichtigung der Belange des Einzelnen. Wo „Integrationsfestigkeit“ vertreten wird, sollte es weniger um Entscheidungsverfahren als wiederum um Inhalte gehen. $\mathrm{Zu}$ diesen gehören vor allem die rationale Erfassung menschlichen Handelns und individueller Verantwortung durch die im allgemeinen Teil des Strafrechts ausgearbeiteten Strukturen. Sie müssen fortentwickelt werden, während das europäische Strafrecht grundsätzlich nicht hinter das in den nationalen Rechtsordnungen bereits Erreichte zurückfallen darf. Zudem dürfen Strafdrohungen auch im Unionsrecht nur als ultima ratio und nur dort, wo sie ein verhältnismäßiges Mittel sind, eingesetzt werden; es greift zu weit, Strafrecht allgemein „zur wirksamen Durchführung der Politik der Union“ einzusetzen (auch wenn „Politik“ im Sprachgebrauch des europäischen Primärrechts eine besondere Bedeutung besitzt).

Die hier bislang vorgetragenen Überlegungen bezogen sich im Wesentlichen auf das System der strafrechtlichen Zurechnungs- und Verhaltensregeln. Oft könnte man sich die gleichen Regeln mit anderen Sanktionen und teils auch auf andere Weise als mittels Sanktionen zwangsbewehrt vorstellen. Der individuelle Vorwurf der Rechtsverletzung charakterisiert das Strafrecht viel besser als die Strafe. Deshalb ist es richtig, dass die strafrechtliche Argumentation vielfach unabhängig von der Sanktions- 
folge geführt wird. Tatsächlich beruhen Fortschritte nicht selten gerade darauf, dass die Unabhängigkeit einer dogmatischen Struktur von der Rechtsfolge erkannt und so eine "reine“ Fassung der tatbestandlichen Voraussetzungen möglich wurde. Inspiriert durch dabei erzielte Erfolge untersuchte und untersucht die Strafrechtswissenschaft von der Sanktion unabhängige dogmatische Strukturen wohl sogar bevorzugt. Auch das hat seine Berechtigung, öffnet aber zugleich Flanken. Es ist nicht nur so, dass die Beschäftigung mit den Sanktionen zum Gegenstandsbereich des Strafrechts gehört und sozusagen „aus Gründen der Vollständigkeit“ nicht vernachlässigt werden darf. Zentrale Probleme des Strafrechts können überhaupt nur unter Bezug auf die Androhung der Sanktion, ihre Zumessung und ihre Vollstreckung behandelt werden.

Mario Bachmann und Ferdinand Goeck (Köln) setzten sich in ihrem gemeinsamen Referat damit auseinander, wie die grundrechtsdogmatische und strafrechtliche Frage der Verhältnismäßigkeit einer Strafdrohung und die Verhältnismäßigkeit und Schuldangemessenheit der zuzumessenden und zu vollstreckenden Strafe empirisch zu fundieren wäre. Im bisherigen Stand der Wirkungsforschung verfüge man zwar über einige vielversprechende Ansätze, aber noch nicht über den angestrebten Überblick. Insbesondere bestehende Rechtslagen und die Schwere der Eingriffe in die Interessen der betroffenen Personen geben recht geringen Spielraum, um die Aussagekraft der Ergebnisse empirischer Studien durch die Herstellung geeigneter Testbedingungen zu fördern.

Dennoch darf man sich den Grundfragen legitimen Strafrechts nicht verschließen. $\mathrm{Zu}$ ihnen gehört insbesondere die stete Suche nach Sanktionen, die gleichzeitig zum Schutz der Rechtsgüter effektiver und für den Betroffenen milder sind als die aktuell verwendeten. Zu ihnen gehört ebenso die Suche nach Kriterien dafür, wann der Einsatz strafrechtlicher Sanktionen gerechtfertigt ist und wo die Rechtsordnung mit anderen Mitteln zu reagieren hat. Mit der Entscheidungsprärogative des demokratischen Gesetzgebers mag man sich in Ermangelung inhaltlicher Antworten behelfen, muss aber auch fragen, auf wie ungesicherter Basis gesetzgeberische Entscheidungen politisches Wunschdenken verfolgen dürfen. Es steht zu erwarten, dass inhaltliche Antworten hier immer Prämissen (und Modelle) voraussetzen werden, die ihrerseits keine absolute Geltung beanspruchen können. Deren Diskussion und Setzung darf dem demokratischen Prozess dauerhaft nicht entzogen werden. Eine rationale und informierte Entscheidung kann aber auch der demokratische Gesetzgeber nur treffen, wenn die bislang noch bei weitem nicht hinreichend ausgearbeiteten Zusammenhänge zwischen solchen Prämissen und ihren Konsequenzen sowohl empirisch als auch dogmatisch bzw. philosophisch eingehender untersucht werden.

Die Tagung war ein Erfolg. Viele am Strafrecht interessierte junge Rechtswissenschaftler kamen - gerade nicht nur Habilitanden, sondern auch Doktoranden und 
einige Studierende. Es wurde ausgiebig in großer Runde und in kleinen Gruppen diskutiert. Die Vorträge boten etliche ausgearbeitete Gedanken, dem Zuhörer aber auch reichlich Gelegenheit, in der eigenen Ordnung der Gedanken Baustellenschilder aufzustellen. Wer verhindert war, wird im Tagungsband zu dieser Tagungsreihe bei Nomos nachlesen können, wie insbesondere das Schuldprinzip, der Verhältnismäßigkeitsgrundsatz und die Regel nulla poena sine lege einerseits in der „Architektur des Verfassungsrechts“ ehrwürdige Schlösser wurden, andererseits aber doch so karg ausgestattet blieben, dass sie ihre volle Funktion erst gewinnen, wenn das Strafrecht sie für seinen Bereich selbst möbliert. Zum entsprechenden Live-Erlebnis werden im kommenden Jahr Kollegen aus Würzburg und Frankfurt am Main an die Goethe-Universität einladen. 